\title{
Le cheveu : \\ des cordes et de la physique
}

Yves Pomeau ${ }^{(1)}$ (pomeau@|ps.ens.fr) et Basile Audoly(2) (basile.audoly@upmc.fr)

(1) Department of Mathematics, University of Arizona, Tucson AZ 85721, USA.

(2) Institut d'Alembert, CNRS et Université Pierre et Marie Curie, France.

Cet article présente un

exemple de l'effet de l'élasticité

d'un solide sur la forme

géométrique qu'il prend

lorsqu'on lui applique

des contraintes : la théorie

mécanique du cheveu.

Le texte souligne, par ailleurs,

le rôle de l'introduction de

méthodes mathématiques,

très générales, en mécanique

du solide.

L'article est un exposé succinct du chapitre 8 du récent ouvrage

"Elasticity and Geometry -

From hair curls to the non-

linear response of shells",

dont nous présentons

page 13, une recension

d'Étienne Guyon.

Remerciements

Le travail décrit ici a été réalisé en collaboration avec Florence Bertails-Descoubes (équipe BiPop de l'INRLA Rhône-Alpes). Les auteurs remercient L'Oréal Recherche, et particulièrement J.-L. Lévéque et B. Querleux, pour les nombreux échanges scientifiques autour de ces questions.

\section{Les cheveux et comment les décrire}

Le cheveu humain est un bon exemple de système mécanique simple à comportement complexe. Sa longueur étant bien supérieure à son diamètre, il peut être décrit par une théorie inspirée de celle de l'Elastica d'Euler, dont les principes sont rappelés plus loin. Ceci conduit à des solutions intéressantes de ce problème de corde. Nous emploierons le mot "corde " au lieu de "tige " ou "poutre ", peut-être plus familiers dans le contexte de la mécanique, mais qui ne paraissent pas très heureux ici. Cette corde étant tendue par son propre poids, sa tension n'est pas uniforme, contrairement au cas d'une corde de piano ou de violon. Une autre particularité " mécanique » du cheveu est qu'il est encastré à une extrémité, au niveau du cuir chevelu, et libre à l'autre. Outre son intérêt comme sujet scientifique, le cheveu, pour son aspect et sa dynamique, est au centre de plusieurs industries. On pense naturellement à la cosmétique, bien représentée dans notre pays, mais aussi à l'industrie de l'image virtuelle (calculée). Jusqu'à tout récemment il n'existait pas de bonne méthode pour simuler de manière réaliste des chevelures : les héroïnes des jeux vidéo et des films en image de synthèse n'avaient que des tresses uniques, relativement faciles à modéliser dans leurs mouvements. Maintenant leurs belles chevelures blondes flottantes peuvent enfin suivre bonds et virevoltes, grâce à la modélisation de la mécanique du cheveu à partir des lois physiques du mouvement d'une corde élastique dont nous allons exposer les principes.

Nous nous étions posés il y a déjà plusieurs années [1] la question de la possibilité de décrire dans un cadre unique les différentes chevelures présentes naturellement : cheveux raides, souples, bouclés, etc. Le but était de réduire les différentes apparences (excluant la couleur) à des changements quantitatifs de quelques paramètres dans un modèle unique. Nous allons en expliquer les principes en nous limitant à un cheveu isolé réduit à une courbe plane, deux limitations qui peuvent être levées.

\section{Qu'est-ce qui donne sa forme à un cheveu?}

Ces cheveux idéaux peuvent être caractérisés par deux paramètres sans dimension, avec des formes en bon accord avec l'observation de la grande diversité des chevelures naturelles. Les équations correspondantes sont inspirées de celles écrites par Euler il y a bientôt 250 ans pour le problème de l'Elastica : Euler s'était posé la question de la stabilité d'une tige élastique verticale soumise à 

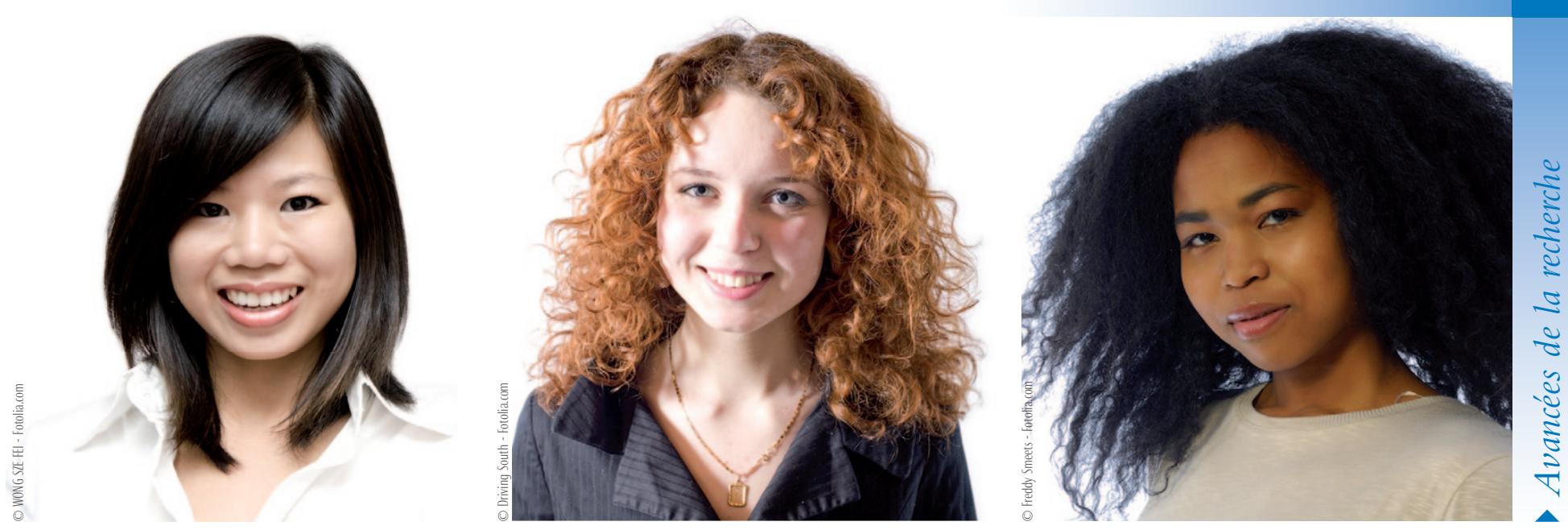

Les trois grands types de cheveux dans le monde. À gauche : cheveux asiatiques (généralement droits et de section ronde) ; à droite : cheveux africains (crépus et de section elliptique) ; au centre : cheveux caucasiens (intermédiaires entre les deux catégories précédentes).

un poids placé à son extrémité supérieure. Il avait montré l'existence d'un poids limite au-delà duquel la configuration minimisant l'énergie totale (élastique et de gravité) n'était plus la configuration verticale, mais celle qui flambe, c'est-à-dire qui fléchit pour abaisser la masse pesante placée en haut et du même coup diminuer l'énergie totale. Ceci réclame une masse critique : l'énergie de courbure croît par la déformation, ce qui doit être compensé par la diminution de l'énergie gravitationnelle. Ce travail d'Euler a joué un rôle considérable dans le développement des sciences, venant après des principes de minimisation comme celui du chemin optique par Fermat. C'est donc pour l'Elastica qu'Euler a jeté les bases de ce que l'on appelle les principes variationnels. La généralisation de ces principes sous-tend une bonne partie de la physique théorique contemporaine, en partie grâce à l'observation due à Lagrange que les lois de la mécanique newtonienne résultent d'une minimisation de l'action.

On considère le cheveu comme une courbe plane inextensible encastrée à une extrémité, et pouvant éventuellement se recouper. On repère le point courant le long de cette courbe par son abscisse curviligne $s$ (fig. 1). Précisément, $s$ est la longueur depuis la racine, longueur qui est presque insensible aux déformations que l'on va considérer, une conséquence assez subtile du grand élancement de cette tige. Le cheveu a une courbure naturelle constante $\kappa_{0}$, alors que dans son état présent (soit après action de la gravité) sa courbure vaut $\kappa(s)$. L'origine de cette courbure tient dans le processus biologique de croissance, très rapide par rapport aux autres processus de croissance cellulaire. Cette courbure naturelle est programmée génétiquement, et il est difficile (mais pas impossible) d'en changer le résultat, soit faire boucler des cheveux raides ou

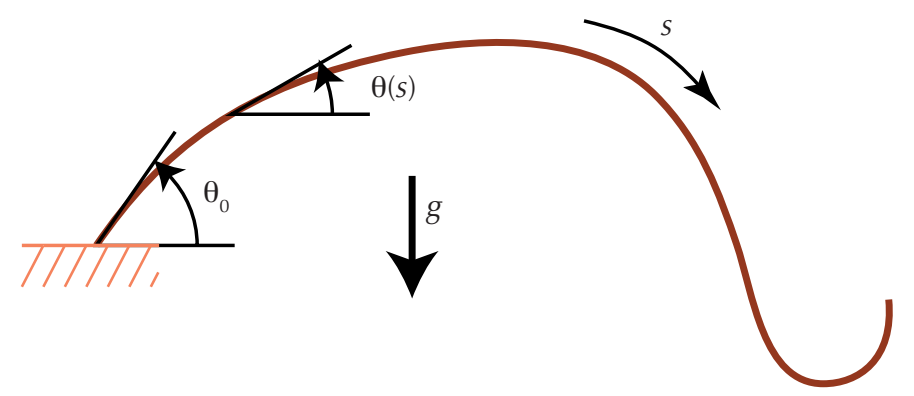

1. Géométrie d'un cheveu plan inextensible. défriser les cheveux trop bouclés, une source de revenus considérables pour l'industrie cosmétique. On suppose que la configuration du cheveu minimise une énergie totale, somme de son énergie potentielle dans le champ de gravité et de son énergie élastique. Cette dernière est proportionnelle au carré de l'écart de la courbure $\kappa(\mathrm{s})$ à sa valeur naturelle $\kappa_{0}$. Pour une forme arbitraire, l'énergie totale vaut :

$$
\varepsilon=\int_{0}^{L} d s\left[\frac{B}{2}\left(\kappa(s)-\kappa_{0}\right)^{2}+\rho g S z(s)\right]
$$

où $B$ est le module de flexion du cheveu, proportionnel à son module d'Young s'il est homogène (à remplacer par une sorte de moyenne de ce module si la section n'est pas de propriétés uniformes) et à la puissance quatrième du rayon du cheveu, $z(s)$ est la coordonnée verticale et $\rho g S z(s)$ ds l'énergie gravitationnelle de l'élément de longueur ds dans le champ vertical d'accélération $g$, $S$ la section du cheveu, $\rho$ la masse volumique et $L$ la longueur totale du cheveu. Notons que ce module $B$ ne dépend pas du module de Poisson. Le module d'Young peut être vu comme une sorte de compressibilité du solide constituant le cheveu. Hors le terme de courbure naturelle, cette énergie est bien celle considérée par Euler pour l'Elastica.

La forme du cheveu résulte de deux effets antagonistes : d'un côté la gravité tend à tirer le cheveu vers le bas; de l'autre, la courbure naturelle constante tend à imposer une forme en boucle circulaire. La minimisation de l'énergie totale permet de trouver la configuration d'équilibre du cheveu soumis à cette double influence. Il existe une dernière contrainte, la direction imprimée à la racine du cheveu par l'encastrement dans le cuir chevelu. On mesure l'orientation par l'angle avec l'horizontale, $\theta(s)$, qui vaut donc $\theta_{0}$, une valeur imposée, à la racine pour $s=0$. La géométrie plane nous dit que $\kappa(s)=\mathrm{d} \theta / \mathrm{d} s$ et $z(s)=\int_{0}^{\mathcal{s}} \mathrm{d} s^{\prime} \sin \theta\left(s^{\prime}\right)$ (fig. 1). Nous allons écrire l'énergie potentielle du cheveu en fonction de sa forme, voir l'équation (2). Pour ce faire, nous allons paramétrer la forme du cheveu au moyen de la fonction $\theta(s)$. Supposant connue $\theta(s)$ et la position du point d'encastrement, il suffit en effet d'intégrer les équations $x^{\prime}(s)=\cos \theta(s)$ et $z^{\prime}(s)=\sin \theta(s)$ pour remonter à la forme dans le plan du cheveu. Nous reformulerons ensuite le problème au moyen de quantités adimensionnées. La minimisation de l'énergie nous conduira aux équations (4) et (5) pour la fonction $\theta(s)$ 
On redéfinit les longueurs en prenant $L$ comme unité. Ce qui donne $\bar{s}=s / L$, qui varie entre 0 (racine) et 1 (extrémité libre). De même $\bar{\kappa}(\bar{s})=L \kappa(s)$ et $\bar{z}=z / L$. Utilisant ces grandeurs réduites, on trouve pour l'énergie :

$$
\varepsilon_{\text {hair }}=\frac{B}{L} \int_{0}^{L} d \bar{s}\left[\frac{1}{2}(\bar{\kappa}(\bar{s})-\alpha)^{2}+\frac{\bar{z}(\bar{s})}{\beta}\right]
$$

écriture qui fait apparaître deux nombres sans dimension, $\alpha$ et $\beta$, définis par :

$$
\alpha=L \kappa_{0} \quad \beta=\frac{B}{\rho g S L^{3}}
$$

\section{Cheveux et diagrammes de phase}

La formulation du problème à l'aide de variables adimensionnées a fait apparaitre $\alpha$ et $\beta$, dont on va pouvoir discuter les variations, puisqu'ils peuvent être grands ou petits, et ceci de façon indépendante l'un de l'autre. La forme d'équilibre va dépendre de ces deux paramètres, ainsi que de la valeur $\theta_{0}$ de l'angle à la racine. Les valeurs de $\alpha$ et $\beta$ varient très largement dans les cheveux humains, d'où l'aspect très varié des chevelures naturelles. Le paramètre $\alpha$ est le rapport de la longueur totale au rayon de courbure naturel $1 / \kappa_{0}$, c'est aussi $2 \pi$ fois le nombre de boucles d'un cheveu en l'absence de gravité. Si $\alpha$ est grand, le cheveu est bouclé. Si au contraire il est faible, le cheveu est faiblement bouclé, presque rectiligne dans son état naturel. L'autre paramètre, $\beta$, détermine l'effet relatif de la gravité par rapport à la raideur élastique : si $\beta$ est petit, alors la gravité est importante et le cheveu tend à tomber verticalement. Si $\beta$ est grand, le cheveu tend à suivre sa forme naturelle, bouclée ou lisse. En fait, les choses sont plus compliquées et exigent de regarder ce qui se passe dans un plan à deux coordonnées, les valeurs de $\alpha$ et $\beta$. Pour $\beta$ et $\alpha$ petits (effet important de la gravité et cheveu raide), le cheveu est droit et flexible, il tombe presque verticalement. Pour $\beta$ petit et $\alpha$ grand il va tendre à tomber, mais son extrémité, soumise à un faible poids, va se courber, puisque le rayon naturel des boucles est très inférieur à la longueur totale. C'est ce que l'on appelle des " anglaises ", sans doute parce que ces boucles « d'extrémité » étaient très appréciées de l'aristocratie anglaise du $19^{\text {e }}$ siècle et souvent reproduites dans des portraits féminins comme ceux de Winterhalter. Le plus grand des portraitistes de femmes, Ingres, représentait, lui, ses modèles avec des chevelures très soigneusement arrangées en chignon, à peine visibles. Pour $\beta$ grand et $\alpha$ petit, la gravité joue peu et la courbure naturelle est faible. Le cheveu tend à jaillir tout raide du cuir chevelu. Pour $\alpha$ et $\beta$ grands, la gravité est faible et les cheveux sont bouclés sans retomber, même près de leur racine.

Une discussion plus quantitative à l'aide de l'équation d'équilibre associée à notre problème de minimisation, obtenue par la méthode d'Euler-Lagrange (présentée dans l'encadré de la page 13), permet de caractériser analytiquement le comportement de la solution dans diverses limites, ce qui donne une très bonne idée de la forme des cheveux suivant les valeurs des paramètres. Dans le cas présent, soit pour l'énergie définie en (2), l'équation de la courbe minimisant l'énergie s'écrit :

$$
\theta ”(s)-\frac{1-s}{\beta} \cos \theta(s)=0
$$

avec les conditions aux limites :

$$
\theta(0)=\theta_{0}, \quad \theta^{\prime}(1)=\alpha .
$$

Dans l'équation (4), $s=0$ représente l'extrémité fixée sur le cuir chevelu, et $s=1$ l'extrémité libre. Cette équation non linéaire n'est pas intégrable pour des valeurs arbitraires de $\alpha$ et $\beta$. On peut caractériser analytiquement le comportement de la solution dans diverses limites, ce qui donne une très bonne idée de la forme des cheveux suivant les valeurs des paramètres. En passant, cette analyse fournit un exemple simple (pédagogique ?) de ce que l'on appelle des développements raccordés, soit un problème où, suivant le domaine considéré, différentes approximations s'appliquent pour une même équation, approximations à raccorder aux frontières entre domaines. Ces idées importantes sont peu enseignées chez nous, contrairement à ce qu'il se passe chez nos voisins britanniques. Nous renvoyons à partir d'ici à notre livre [2], où les détails de l'analyse sont exposés. La figure 2 donne une image des différents régimes rencontrés suivant les valeurs des paramètres $\alpha$ et $\beta$. Contentons-nous de quelques détails sur le cas le plus facile, soit celui d'un cheveu naturellement très bouclé et léger. C'est la limite $\beta \rightarrow \infty$. L'équation (4) se réduit à $\theta$ " $(s)=0$, avec la solution $\theta(s)=\theta_{0}+\alpha s$, soit un arc de cercle de courbure $\kappa_{0}$ constante. Cette solution peut être affinée en considérant les termes d'ordre supérieur dans le petit paramètre $1 / \beta$, ce qui permet de trouver de combien le cheveu tombe dans le champ de pesanteur.

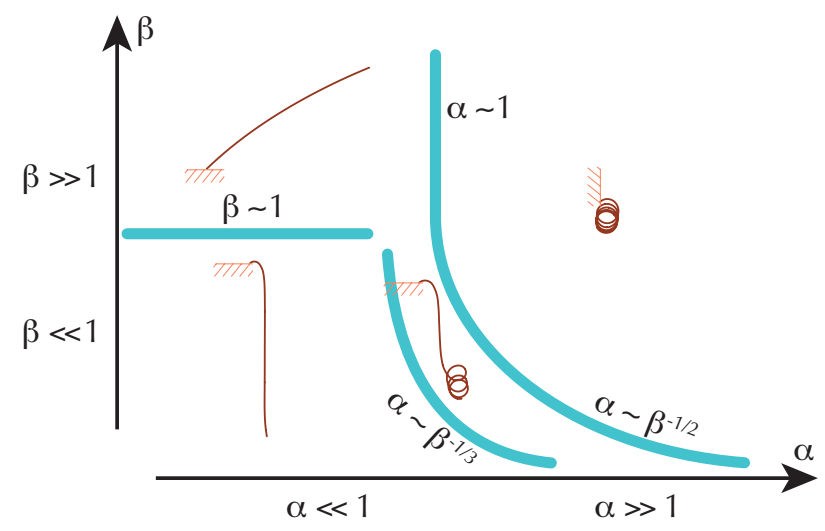

2. Classification des formes de cheveux selon la valeur des paramètres réduits $\alpha$ mesurant la courbure spontanée et $\beta$ mesurant la raideur rapportée au poids. Les rectangles hachurés représentent l'encastrement.

\section{Dynamique des cheveux}

Nous avons fait allusion, au début, aux longues chevelures des hérö̈nes de jeux vidéos sautant et bondissant. Nous allons donc dire quelques mots de la dynamique des cheveux, conçue dans le même esprit, c'est-à-dire à partir des principes de la mécanique des cordes. Bien sûr, l'inclusion d'effets dynamiques est un problème beaucoup plus difficile que celui de la configuration d'équilibre. Par le génie de Lagrange, on sait transformer ce problème dynamique en la minimisation d'une fonctionnelle de l'histoire du mouvement, l'action. Cette minimisation conduit à une équation dynamique formellement proche de celle de la condition d'équilibre, une équation de la forme :

$$
\partial \mathbf{F}(s, t) / \partial s+\mathbf{p}(s, t)=\rho S \ddot{\mathbf{r}}(s, t),
$$

où $t$ est la variable temps, $\rho S$ la masse de la corde par unité de longueur et $\ddot{\mathbf{r}}$ l'accélération locale. Sans entrer dans le détail, ce qui exigerait de passer de la formulation intrinsèque (sans repère défini) à des coordonnées cartésiennes, indiquons juste que dans 
cette équation $\mathbf{F}(s, t)$ représente les forces (internes) nées de la courbure, $\mathbf{p}(s, t)$ la force de pesanteur, $\ddot{\mathbf{r}}(s, t)$, dans le second membre étant l'accélération locale. L'équation d'équilibre (sans mouvement donc) est retrouvée en mettant à zéro le second membre de cette équation du mouvement. Le terme d'accélération change profondément la structure de l'équation par rapport au cas purement statique. Par exemple, le terme de force interne $\mathbf{F}(s, t)$ ne peut être déterminé à l'avance puisqu'il se couple à l'accélération. Ces équations constituent un système " raide » dans la terminologie mathématique. En étendant les méthodes développées pour le cas statique, on a pu trouver des méthodes de calculs pour ces équations du mouvement.

\section{Conclusion}

La mécanique du cheveu humain fournit un exemple d'utilisation des concepts de la théorie des cordes élastiques. L'analyse nous a conduit à appliquer une panoplie de méthodes permettant de caractériser un ensemble assez varié de comportements possibles. Un des résultats les plus remarquables est sans doute la classification des différents aspects à l'aide de modèles simples à quelques paramètres au sens physique clairement défini. C'est aussi une preuve qu'un effort de compréhension rationnelle peut rencontrer le succès dans des domaines a priori éloignés du champ traditionnel des sciences dites dures. Ceci pourrait être utile à méditer à une époque où ces sciences dures peinent tant à attirer les jeunes générations.

\section{Le principe d'Euler-Lagrange}

La méthode d'Euler-Lagrange généralise la condition de gradient nul associée à la stationnarité d'une fonction dépendant de variables discrètes, au cas d'une fonctionnelle, c'est-à-dire une fonction dépendant elle-même d'une fonction inconnue. Des exemples classiques de telles fonctionnelles sont le chemin optique, fonction de la trajectoire d'un rayon lumineux, l'action, fonction de la trajectoire d'un point matériel, ou encore l'énergie d'une poutre, fonction de sa déformée. La condition de stationnarité d'Euler-Lagrange, satisfaite quand la fonctionnelle est extrémale, s'écrit sous la forme d'une équation différentielle pour la fonction inconnue. Appliqué à l'optique, ce formalisme fournit l'équation différentielle pour la trajectoire d'un rayon lumineux dans un milieu d'indice variable, ce dernier réalisant un minimum du chemin optique par le principe de Fermat. Dans le cas des poutres qui nous intéressent ici, et partant de la fonctionnelle d'énergie (2) et d'une condition aux limites cinématique $\theta(0)=\theta_{0}$, le formalisme d'Euler-Lagrange fournit l'équation (4) caractérisant les solutions d'équilibre ainsi que la deuxième condition aux limites de l'équation (5), $\theta^{\prime}(1)=\alpha$, appelée condition aux limites naturelle.

\section{Elasticity and Geometry - From hair curls to the non-linear response of shells}

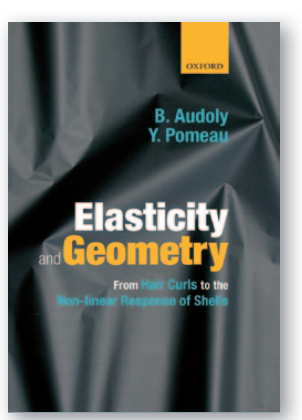

\author{
Basile Audoly \\ et Yves Pomeau \\ (0xford University Press, 2010, \\ 600 p., 75,00 f).
}

Les effets de l'élasticité des solides sur les formes géométriques qu'ils prennent lorsqu'on leur applique des contraintes sont omniprésents. L'ouvrage de Basile Audoly et Yves Pomeau est une contribution originale sur ce thème, et constitue un ouvrage de référence avancée essentiel. Superbement illustré, il donne une présentation claire de cette réponse élastique d'un solide, tout particulièrement pour des géométries de structures élancées telles que une ou deux dimensions sont petites devant la taille globale (tiges, feuilles, coques). Chaque chapitre bénéficie d'une présentation, d'illustrations et d'une conclusion permettant une lecture aisée pour un traité aussi avancé.

Dans l'article ci-dessus, ils nous présentent un exemple de ce travail considérable : l'étude de l'élasticité d'un cheveu ; cet article souligne par ailleurs le rôle de cette mécanique du solide dans l'introduction de méthodes mathématiques très générales.

Néanmoins, ce sujet ne fait pas partie de la formation générale du physicien (qui serait souvent bien en peine de calculer la rigidité d'un ressort spiral à partir de la donnée de ses caractéristiques élastiques et de sa géométrie !). Ce domaine est à peine évoqué dans l'enseignement classique, sauf chez les géophysiciens; de plus, il n'y a pas actuellement (à part le volume Théorie de l'élasticité de Landau et Lifchitz) d'ouvrage de base de physique ou de mécanique physique sur ces sujets. Enfin, un tel ouvrage rend justice à l'intérêt de nombreux physiciens pour des problèmes de mécanique des milieux continus (cet intérêt est apparu, par exemple, dans les journées d'études communes entre la Société Française de Physique et l'Association Française de Mécanique).

Étienne Guyon PMMH, ESPCI

\section{Références}

1 F. Bertails, B. Audoly, B. Querleux, M.-P. Cani, F. Leroy et J.-L. Lévêque,

"Modélisation de coiffures naturelles à partir des propriétés physiques du cheveu ", 18 $8^{\text {es }}$ Journées de l'Association Française d'Informatique Graphique (AFIG 2005, Strasbourg, 28-30 novembre 2005), http://hal.inria.fr/docs/00/51/94/70/PDF/BertailsAFlG05.pdf

2• B. Audoly et Y. Pomeau, Elasticity and Geometry - From hair curls to the non-linear response of shells, Oxford University Press (2010). 